\title{
Tranexamic acid: When is enough (data) enough?
}

\author{
Fiona E. Ralley, MBChB
}

Received: 9 July 2015/ Accepted: 10 July 2015/Published online: 26 August 2015

(c) Canadian Anesthesiologists' Society 2015

Tranexamic acid (TXA) is a synthetic analog of the amino acid lysine which exerts its effect by binding with the lysine-binding sites on the plasminogen molecule. It reversibly and competitively reduces the affinity of plasminogen binding with fibrin, decreases the activation of plasminogen to plasmin, and reduces the local degradation of fibrin by plasmin. Since its development in the early 1960s, TXA has been proven to be a remarkably effective drug in reducing perioperative blood loss and therefore the requirement for transfusion. ${ }^{1,2}$ Nowhere has this been more apparent than in orthopedic surgery, especially in patients undergoing major joint replacement procedures. Until recently, concerns over TXA's theoretical potential to increase postoperative venous thrombotic events (VTE) have unfortunately delayed or even prevented its broader use among arthroplasty patients. Venous thrombotic events remain a concern in arthroplasty patients who are especially vulnerable to thrombotic complications postoperatively.

Initial in vitro studies have shown that a concentration of $100 \mathrm{mg} \cdot \mathrm{L}^{-1}$ was required to produce a $98-100 \%$ reduction in fibrinolytic activity. ${ }^{1}$ Subsequent clinical experience has shown that an $80 \%$ reduction in fibrinolytic activity was sufficient for clinical needs, and this was produced with a TXA concentration of 10 $\mathrm{mg} \cdot \mathrm{L}^{-1} .{ }^{1}$ The optimal dose and route of administration to be used in arthroplasty patients remain unclear. In a recent meta-analysis, 46 randomized controlled trials involving

F. E. Ralley, $\mathrm{MBChB}(\bowtie)$

Department of Anesthesia \& Perioperative Medicine, Schulich School of Medicine \& Dentistry, Western University, University Hospital, Rm. C3-110, 339 Windermere Road,

London, ON N6A 5A5, Canada

e-mail: fralley@uwo.ca
2,925 patients undergoing an orthopedic procedure were identified and included in the analysis. ${ }^{3}$ In 21 studies, TXA was given in doses $<15 \mathrm{mg} \cdot \mathrm{kg}^{-1}$, and in 18 studies, TXA was given in doses $>15 \mathrm{mg} \cdot \mathrm{kg}^{-1}$. A single bolus given preoperatively was used in 20 studies, and repeated boluses were given in 26 studies. When all doses and schedules were pooled, TXA was associated with a mean reduction in total blood loss of $-408 \mathrm{~mL}$ (95\% confidence interval [CI], -506 to -311 ), leading to a halving of the likelihood of allogeneic blood transfusion (relative risk, $0.51 ; 95 \% \mathrm{CI}$, 0.46 to 0.56 ). Subgroup analysis showed that reductions in transfusion were similar in both single and multiple TXA dosing regimens. In addition, these authors did not observe any statistically significant increases in thromboembolic events. There were similar rates of deep venous thrombosis (DVT) in the TXA and control groups [30 of 1,376 (2.18\%) patients and 26 of $1,313(1.98 \%)$ patients, respectively; relative risk, $1.11 ; 95 \%$ confidence interval, 0.69 to 1.79 ].

Despite the plethora of individual studies and meta-analyses, the routine use of TXA in this at-risk patient population has been limited by what some clinicians consider to be an insufficiency of data. This is mainly due to the small sample size of the majority of studies, thus requiring meta-analyses to characterize the safety and harm of therapy. A recent large retrospective cohort study drawn from administrative health data may have put some of these concerns to rest. ${ }^{4}$ In this study of 872,416 patients who had total hip or knee arthroplasty, multilevel multivariable logistic regression modelling was used to assess the association between intravenous use of TXA and patient outcome. Tranexamic acid was given to 20,051 patients, and results at the end of the seven-year study period showed that the proportion of patients receiving TXA increased from almost $0 \%$ to $11.2 \%$. Use of TXA was associated with halving the likelihood of allogeneic or 
autologous blood transfusion (odds ratio, 0.50; 95\% CI, 0.45 to 0.55 ). More importantly, the study results showed that this treatment benefit was not associated with an increased risk of VTE (odds ratio, 0.86; 95\% CI, 0.59 to 1.25). The large study size allowed for an evaluation of safety concerns regarding the use of TXA, especially the incidence of rare thrombotic events such as deep venous thrombosis or pulmonary embolism. Patients who received TXA actually had a lower incidence of VTE when compared with patients who were not given the drug ( $0.6 \%$ vs. $0.8 \%$, respectively). Moreover, the study population represented "real-world" practice unlike data from most randomized controlled trials where participants are often highly selected.

In this issue of the Journal, Baker et al. ${ }^{5}$ present their data after implementing a universal protocol directing TXA administration in eligible patients undergoing arthroplasty surgery. They also assessed the impact of TXA administration on red blood cell (RBC) transfusion, postoperative hemoglobin, hospital length of stay, and adverse events. They compared 422 patients prior to the implementation of the protocol (use of TXA 45.6\%) with the first 422 patients after implementation of the protocol (use of TXA 95.3\%). Results showed an overall reduction in $\mathrm{RBC}$ transfusion from $8.8 \%$ to $5.2 \%$, representing a change of $3.6 \%$ (95\% CI, 0.1 to 7.0 ). This reduction was more dramatic in patients with preoperative anemia (hemoglobin $<120 \mathrm{~d} \cdot \mathrm{L}^{-1}$ ) where transfusion rates were reduced from $28.6 \%$ to $18.6 \%$. In this study, a $20 \mathrm{mg} \cdot \mathrm{kg}^{-1}$ dose of TXA was used in an attempt to provide a balance between reducing perioperative blood loss and RBC transfusion while limiting the incidence of potential adverse events. Again, a more "real-world" "all-comers" approach was used where there were only a few absolute contraindications to the use of TXA (e.g., patients with a recent history of myocardial infarction, stroke, DVT, pulmonary embolism, seizure disorder, renal insufficiency, placement of a bare metal stent within six months or a drug-eluting stent within 12 months, history of hypercoagulability or an inability to receive standard DVT prophylaxis). Despite these relative contraindications, 14 patients (including nine patients with known atrial fibrillation, two patients with a remote history of stroke, one patient with a remote history of pulmonary embolus, and two patients with a history of coronary artery disease including one patient who had received a stent 15 months prior to surgery) received intraoperative TXA without any adverse outcomes. One minor limitation of the study might be that no formal transfusion algorithm was used in either group of patients. Nevertheless, again this study better represents a "real-world" situation. Obviously, the study is also somewhat limited by the retrospective nature of the data collection; however, it would appear that the authors went to significant lengths to ensure complete and accurate data. A short study time period also helped reduce any confounding effects of changes in patient's management. One surprising finding in this study was the incidence of acute kidney injury (AKI) as defined by the RIFLE classification. ${ }^{6}$ Acute kidney injury was seen in eight of 422 patients before implementation of the TXA protocol and in 16 of 422 patients following the protocol. This difference did not reach statistical significance $(P=0.147)$; moreover, it would appear that either the use of TXA or RBC transfusion was associated with the risk of developing AKI postoperatively. The serum creatinine returned to baseline values in all patients within a mean of two days. The importance of these observations still needs to be assessed.

So have we reached saturation point yet? In 2015 alone, over 39 articles on the topic of tranexamic acid and arthroplasty have already been recorded in PubMed, and nearly a hundred articles appeared in 2014. In the MerriamWebster dictionary, enough is defined as "occurring in such quantity, quality, or scope as to fully meet demands, needs, or expectations", and data is defined as "facts or information used usually to calculate, analyze or plan something". If we place these two definitions together, does it not suggest that perhaps we have the answer? Certainly, there is an overabundance of evidence that TXA reduces blood loss in arthroplasty patients. It would now appear that we have the data to support the position that TXA does not increase (or decrease) the VTE risk associated with this type of surgery. In addition, the risks associated with a blood transfusion are also well documented. Should we not use these facts and do what is best, i.e., weigh the risks and benefits for each case and decide if the use of TXA is warranted for this patient? The introduction of protocols as implemented by Baker et al. will go a long way to ensure that this currently underused drug will be given to the right patient, in the right dose, and at the right time.

\section{Acide tranexamique : quand assez (de données), c'est assez?}

L'acide tranéxamique (TXA) est un analogue de synthèse de la lysine, un acide aminé qui agit en se liant aux sites de fixation de la lysine sur les molécules de plasminogène. Il diminue de façon réversible et compétitive l'affinité de liaison du plasminogène pour la fibrine, diminue l'activation du plasminogène en plasmine, et diminue la dégradation locale de la fibrine par la 
plasmine. Depuis son développement au début des années 1960, on a montré que le TXA était un médicament remarquablement efficace pour réduire les pertes sanguines périopératoires et donc le recours aux transfusions. ${ }^{1,2}$ Cela n'a jamais été aussi évident qu'en chirurgie orthopédique, en particulier chez les patients subissant des interventions majeures d'arthroplasties. Jusqu'à récemment, les questions concernant le risque théorique de voir le TXA augmenter le nombre d'évènements thrombotiques veineux (ETV) postopératoires ont malheureusement retardé, voire empêché sont utilisation plus large chez les patients subissant une arthroplastie. Les évènements thrombotiques veineux restent une préoccupation chez les patients subissant une arthroplastie et qui sont particulièrement exposés aux complications thrombotiques en postopératoire.

Les études initiales in vitro ont montré qu'une concentration de $100 \mathrm{mg} \cdot \mathrm{L}^{-1}$ était nécessaire pour entraîner une réduction de l'activité fibrinolytique de $98 \%$ à $100 \%{ }^{1}$ L'expérience clinique ultérieure a montré qu'une réduction de l'activité fibrinolytique de $80 \%$ était suffisante pour répondre aux besoins cliniques et que cela était atteint avec une concentration de TXA de $10 \mathrm{mg} \cdot \mathrm{L}^{-1} \cdot{ }^{1}$ La dose et la voie d'administration optimales à utiliser chez les patients subissant une arthroplastie restent à préciser. Récemment, 46 essais randomisés et contrôlés ayant inclus 2925 patients subissant une intervention orthopédique ont été identifiés et inclus dans une méta-analyse. ${ }^{3}$ Dans 21 études, le TXA a été administré à des doses $<15 \mathrm{mg} \cdot \mathrm{kg}^{-1}$ et dans 18 études, il a été administré à des doses $>15 \mathrm{mg} \cdot \mathrm{kg}^{-1}$. Un bolus unique a été administré en préopératoire dans 20 études tandis que des bolus répétés étaient administrés dans 26 études. Après regroupement de toutes les doses et conditions d'administration, le TXA a été associé à une réduction moyenne totale des pertes sanguines de $-408 \mathrm{~mL}$ (intervalle de confiance [IC] à $95 \%:-506$ à -311), aboutissant à diminuer de moitié la probabilité d'une transfusion de sang allogène (risque relatif : 0,51 ; IC à $95 \%, 0,46$ à 0,56$)$. Une analyse par sous-groupes a montré que les réductions des transfusions étaient semblables, que les patients aient reçu une seule injection ou plusieurs injections de TXA. De plus, ces auteurs n'ont pas observé d'augmentations statistiquement significatives des évènements thromboemboliques. Les taux de thrombose veineuse profonde (TVP) ont été similaires dans les groupes TXA et contrôle [respectivement : 30/1 $376(2,18 \%)$ patients et 26/1 $313(1,98 \%)$ patients; risque relatif : 1,11 ; IC à $95 \%: 0,69$ à 1,79$]$.

En dépit de la pléthore d'études individuelles et de méta-analyses, l'utilisation régulière du TXA dans cette population à risque a été limitée par ce que certains cliniciens considèrent comme une insuffisance de données. Cela est principalement dû à la petite taille de la majorité des études, et donc à la nécessité de recourir à des méta-analyses pour déterminer l'innocuité ou la nocivité d'un traitement. Une grande étude récente de cohorte rétrospective extraite des données administratives de santé a peut-être permis de répondre à ces préoccupations. ${ }^{4}$ Dans cette étude de 872416 patients ayant subi une arthroplastie totale de genou ou de hanche, un modèle de régression logistique multifactorielle à plusieurs niveaux a été utilisé pour évaluer l'association entre l'administration intraveineuse de TXA et l'évolution des patients. L'acide tranexamique a été administré à 20051 patients et les résultats au terme de la période d'étude de 7 ans ont montré que le pourcentage de patients recevant du TXA est passé de presque $0 \%$ à $11,2 \%$. L'utilisation du TXA a été associée à une réduction de moitié de la probabilité de transfusion sanguine autologue ou allogénique (rapport de cotes : 0,50 ; IC à $95 \%, 0,45$ à 0,55 ). Surtout, les résultats de l'étude ont montré que les avantages de ce traitement n'étaient pas associés à une augmentation du risque d'ETV (rapport de cotes : 0,86; IC à $95 \%, 0,59$ à 1,25). La grande taille de l'étude a permis une évaluation des préoccupations d'innocuité concernant l'utilisation du TXA, notamment l'incidence d'évènements thrombotiques rares tels que la thrombose veineuse profonde ou l'embolie pulmonaire. En fait, les patients ayant reçu du TXA avaient une incidence d'ETV inférieur à celle des patients n'ayant pas reçu le médicament (respectivement, $0,6 \%$ contre $0,8 \%$ ). La population de l'étude représentait les conditions réelles d'exercice contrairement aux données de la majorité des études randomisées et contrôlées dans lesquelles les patients font l'objet d'une sélection rigoureuse.

Dans ce numéro du Journal, Baker et coll. ${ }^{5}$ présentent leurs données après l'implémentation d'un protocole universel orientant l'administration de TXA à des patients admissibles subissant une arthroplastie. Ils ont également analysé l'impact de l'administration de TXA sur les transfusions de globules rouges, l'hémoglobine postopératoire, la durée de l'hospitalisation et les évènements indésirables. Ils ont comparé 422 patients avant la mise en œuvre du protocole (utilisation de TXA : $45,6 \%$ ) avec les 422 premiers patients suivant la mise en œuvre du protocole (utilisation de TXA : 95,3\%). Les résultats ont montré une réduction globale des transfusions de globules rouges de $8,8 \%$ à $5,2 \%$, soit une différence de $3,6 \%$ (IC à $95 \%: 0,1$ à 7,0). Cette baisse a été encore plus impressionnante chez les patients présentant une anémie préopératoire (hémoglobine $<120 \mathrm{dL}^{-1}$ ) pour lesquels les taux de transfusion sont passés de 28,6\% à 18,6\%. Dans cette étude, une dose de TXA de $20 \mathrm{mg} \cdot \mathrm{kg}^{-1}$ a été utilisée pour tenter de trouver un équilibre entre la réduction périopératoire des pertes sanguines et la transfusion de globules rouges tout en limitant l'incidence d'évènements indésirables possibles. Ici encore, une perspective de 
«monde réel » et de «patients tout-venant» a été utilisée quand il n'y avait que peu de contre-indications absolues à l'utilisation du TXA (par exemple, patients ayant récemment présenté : infarctus du myocarde, AVC, TVP, embolie pulmonaire, crises convulsives, insuffisance rénale, mise en place d'un tuteur coronarien en métal nu dans les six derniers mois ou un tuteur coronarien à libération de produit actif dans les 12 derniers mois, des antécédents d'hypercoagulabilité, ou patients ne pouvant pas recevoir une prophylaxie standard de la TVP). En dépit de contre-indications relatives, 14 patients (neuf patients avaient une fibrillation auriculaire connue, deux patients avaient des antécédents anciens d'AVC, un patient avait un antécédent lointain d'embolie pulmonaire et deux patients avaient un historique de coronaropathie l'un d'entre eux ayant reçu un tuteur coronarien 15 mois avant l'intervention chirurgicale) ont reçu du TXA en peropératoire sans aucun évènement préjudiciable. Une restriction mineure de cette étude pourrait être le fait qu'aucun algorithme formel de transfusion n'a été utilisé pour aucun des groupes de patients. Néanmoins, cette étude représente de nouveau une situation encore meilleure de «monde réel ». Évidemment, l'étude est quelque peu limitée par la nature rétrospective de la collecte des données, mais il semble que les auteurs se sont donnés beaucoup de mal pour garantir la collecte de données exactes et complètes. La courte période d'étude a également contribué à réduire tous les effets confondants des changements dans la gestion des patients. Une constatation surprenante de cette étude a été l'incidence des lésions rénales aiguës (LRA) telles que définies par la classification RIFLE. $^{6}$ Une lésion rénale aiguë a été observée chez huit des 422 patients avant la mise en œuvre du protocole TXA et chez 16 patients sur 422 après l'implémentation du protocole. Cette différence n'a pas atteint la signification statistique $(P=0,147)$; il semblerait en outre que l'utilisation de TXA ou la transfusion de globules rouges a été associée au risque de développement d'une LRA postopératoire. Les taux de créatinine sérique sont revenus à leur valeur initiale chez tous les patients en deux jours, en moyenne, L'importance de ces observations reste encore à évaluer.

Alors avons-nous atteint un point de saturation? Rien qu'en 2015, plus de 39 articles sur le sujet de l'acide tranexamique et de l'arthroplastie ont déjà été enregistrés sur PubMed et près d'une centaine d'articles a été publiée en 2014. Dans le dictionnaire Merriam-Webster, « assez » est défini de la manière suivante: "survient en telles quantité, qualité ou étendue que les demandes, besoins ou attentes sont pleinement satisfaits », et « données » sont ainsi définies «faits ou renseignements utilisés habituellement pour calculer, analyser ou planifier quelque chose ». Si nous plaçons ces deux définitions côte à côte, ne suggèrent-elles pas que nous avons peut-être la réponse? Il existe indiscutablement une surabondance de preuves montrant que le TXA diminue les pertes sanguines chez les patients subissant une arthroplastie. Il semble aussi que nous pouvons maintenant affirmer que le TXA n'augmente ni ne diminue le risque d'ETV associé à ce type d'intervention chirurgicale. De plus, les risques liés à une transfusion sanguine sont aussi bien documentés. Devons-nous utiliser ces faits et faire ce qu'il y a de mieux, c'est-à-dire, peser les risques et avantages dans chaque cas et décider si l'utilisation du TXA est justifiée pour un patient donné? L'introduction de protocoles, tels que ceux mis en œuvre par Baker et coll. ira loin pour s'assurer que ce médicament sous-utilisé soit donné aux bons patients, à la bonne dose et au bon moment.

Conflicts of interest None declared.

Disclosure Director of ONTraC program at LHSC. Member of the ONTraC steering committee.

Conflits d'intérêts Aucun déclaré.

Divulgation des fonctions Directeur du programme ONTraC du LHSC. Membre du comité directeur de l'ONTraC.

\section{References}

1. Andersson L, Nilsson IM, Colleen S, Granstrand B, Merlander B. Role of urokinase and tissue activator in sustaining bleeding and the management thereof with EACA and AMCA. Ann N Y Acad Sci 1968; 146: 642-58.

2. Andersson L, Nilsson IM, Nilehn JE, Hedner U, Granstrand B, Merlander B. Experimental and clinical studies on AMCA, the antifibrinolytically active isomer of p-aminomethyl cyclohexane carboxylic acid. Scand J Haematol 1965; 2: 230-47.

3. Huang $F, W u D, M a G$, Yin $Z$, Wand $Q$. The use of tranexamic acid to reduce blood loss and transfusion in major orthopedic surgery: a meta-analysis. J Surg Res 2014; 186: 318-27.

4. Poeran J, Rasul R, Suzuki S, et al. Tranexamic acid use and postoperative outcomes in patients undergoing total hip or knee arthroplasty in the United States: retrospective analysis of effectiveness and safety. BMJ 2014; 349: g4829.

5. Baker JE, Pavenski K, Razuk AP, et al. Universal tranexamic acid therapy to minimize transfusion for major joint arthroplasty: a retrospective analysis of protocol implementation. Can J Anesth 2015; 62: this issue. DOI:10.1007/s12630-015-0460-6.

6. Acute Dialysis Quality Initiative Workgroup. Acute renal failure definition, outcome measures, animal models, fluid therapy and information technology needs: the Second International Consensus Conference of the Acute Dialysis Quality Initiative (ADQI) Group. Crit Care 2004; 8: R204. 